\title{
Juegos del S. XVI en las clases de Educación Física de Secundaria Sixteenth century games in Secondary Physical Education classes
}

\author{
Julio César Rodríguez de la Cruz \\ IES Santo Tomás de Aquino, Íscar - Valladolid (España)
}

\begin{abstract}
Resumen: A los docentes de la Educación Física nos suele caracterizar nuestro interés por la formación permanente, así como por la creación de nuestros propios recursos didácticos. En esta línea de actuación, nuestro departamento se constituyó en el curso 2003-2004 como Grupo de Trabajo para propiciar un lugar de intercambio y encuentro de ideas y experiencias. De este foro surgió una Unidad Didáctica para la Secundaria con los juegos y concursos de precisión practicados entre finales del siglo XV y mediados del XVI (coincidiendo con el reinado de Isabel I) como principales protagonistas. Además, en esta ocasión en particular, el trabajo específico del área se unió a otro multidisciplinar en aras de procurar un estimulante y enriquecedor vehículo de conocimiento sobre ese momento histórico. Por último, comentar que el producto final alcanzado dio unos frutos extraordinarios con grupos de alumnos con muy baja motivación hacia cualquier tipo de actividad físico y/o deportiva, por lo que su valor se incrementa aún más.
\end{abstract}

Palabras clave: Reina Isabel I. Juegos de precisión. Educación Física. Interdisciplinaridad.

\begin{abstract}
Physical Education teachers are characterized by our interest in both permanent training and elaborating our own didactic resources. Based on this performance, our department took form of a work group during 2003-2004 as a place to share and exchange ideas and experiences. Out of this forum, we created a didactic unit for Secondary Education with games and accuracy contests practised during the late fifteenth and mid sixteenth centuries (while the reign of Elizabeth I) as main topics. Besides, on this particular occasion, our specific subject work involved a multi-disciplinary approach in order to obtain some stimulant and enriching knowledge of that historical moment. Finally, the result that we achieved was successful with a group of students who don't feel at all motivated by any kind of physical activity. Therefore, we value this work highly as an educational tool.

Key words: The queen Elizabeth I. Accuracy games. Physical Education. Syllabus.
\end{abstract}

\section{Justificación}

Con motivo de la conmemoración del V Centenario de la muerte de la reina Isabel la Católica (2004), en la comunidad castellano-leonesa se sucedieron múltiples actos y actividades que pretendían, entre otra serie de cosas, dar a conocer la época y reinado de este personaje histórico.

Parece lógico pensar que nuestra actuación desde la Educación Física, por la carencia en aquel momento de tratados que la sistematizasen tal y como hoy la conocemos, tendrá en el juego el elemento clave en torno al cual girará la misma. Por tanto, en su momento consideramos muy en la línea de este tipo de actividades conmemorativas la realización de una Unidad Didáctica en la que el juego fuese el denominador común e identificador de todas nuestras propuestas pedagógicas, siendo la época del final del reinado de Isabel I el pretexto que da sentido a las actividades planteadas.

Además, ya que durante dicho reinado tuvieron lugar los descubrimientos realizados por Cristóbal Colón, figura ilustre de la que ahora se están celebrando los actos conmemorativos del V centenario de su muerte (1451-1506), resulta evidente que este trabajo didáctico vuelve a tener un especial sentido en nuestras aulas.

Conviene matizar que la Unidad Didáctica que aquí esbozamos se puede consultar completamente desarrollada en una publicación en formato CD que la Junta de Castilla y León, a través de su Consejería de Educación y Cultura, ha editado bajo el título genérico de «Experiencias de Aula VI». Finalmente, en el Cuadro 1 se sintetizan los puntos desarrollados en el artículo.

\section{Metodología de la investigación}

Toda esta experiencia arranca de la inquietud y necesidad de un grupo pequeño de profesores de Educación Física de seguir formándose en su quehacer diario. A partir de una propuesta no oficial del Centro de Formación e Innovación Educativa (CFIE) del que dependía nuestro centro de trabajo, decidimos crear un Grupo de Trabajo para investigar,

Fecha recepción: 09-07-07 - Fecha envío revisores: 09-07-07 - Fecha de aceptación: 12-11-07 Correspondencia: Julio César Rodríguez de la Cruz

C/ Nueva, $6,2^{\circ} \mathrm{D}$

47100, Tordesillas - Valladolid (España)

E-mail: juliocesarrodri@mixmail.com crear y aplicar en nuestras clases juegos y prácticas físico-deportivas que pudieran haber tenido cabida en el final del siglo XV y comienzo del XVI, coincidiendo con el término del reinado de Isabel I.

Nuestros objetivos estaban claros: diseñar y elaborar una Unidad Didáctica que tuviese a los juegos practicados en ese periodo histórico como principales protagonistas, para su posterior aplicación y desarrollo en nuestras clases de Educación Física en Secundaria.

El segundo paso era situarnos temporal y contextualmente para conocer los posibles juegos y prácticas físicas que entonces había, por lo que comenzamos el trabajo por la investigación bibliográfica.

Somos conocedores que este tipo de investigación conlleva cierto margen de duda sobre las fuentes consultadas (Bernando y Calderero, 2000), pero tampoco es menos cierto que no había otra forma de conocer, al menos someramente, lo que nos interesaba. En todo caso, siempre que podíamos contrastábamos las informaciones que más nos interesaban con otras fuentes. En este sentido, hemos de decir que Internet no supuso la ayuda que hubiéramos deseado.

Una vez tuvimos claros los juegos que eran practicados en aquella época pasamos a contrastarlos con los que de forma más detallada aparecían recogidos en las fuentes de documentación sobre juegos populares tradicionales propios de la comunidad castellano leonesa. Obviamente hay juegos que ya no se practican en la actualidad y, sin embargo, hay otros más actuales que perfectamente tenían cabida en la Unidad Didáctica que pretendíamos diseñar. Para no dispersarnos en las propuestas acordamos que la gran mayoría de los juegos deberían tener un denominador común: la necesidad de demostrar puntería o precisión en el desarrollo del mismo.

A partir de ese momento podemos decir que la metodología de trabajo adquirió tintes de investigación-acción ya que, a partir de propuestas concretas de trabajo, algunos de nosotros hacíamos de observadores externos para luego, en el seno del grupo, reflexionar sobre la propia práctica, corroborando aquello que creíamos acertado, replanteando lo que considerábamos que no estaba totalmente correcto y desechando lo no adecuado.

Por último, con este tipo de metodología conseguimos, entre otras cosas, tal y como señala Blández (2000, 24-27), aumentar nuestra autoestima y motivación profesional, reflexionar sobre nuestra propia práctica, así como investigar para crear nuestros propios materiales. 


\begin{tabular}{|c|c|c|}
\hline & APARTADO & CONTENIDO \\
\hline & $\begin{array}{l}\text { Metodología de la } \\
\text { investigación }\end{array}$ & $\begin{array}{l}\text { Breve exposición de los aspectos metodológicos básicos de la investigación y del } \\
\text { posterior trabajo desarrollado. }\end{array}$ \\
\hline \multirow{9}{*}{ 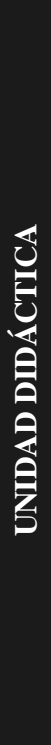 } & Introducción & $\begin{array}{l}\text { Concreción del ámbito de aplicación; Consideraciones previas; Aclaración de la } \\
\text { principal fuente de documentación. }\end{array}$ \\
\hline & $\begin{array}{l}\text { Actividad física de la } \\
\text { época }\end{array}$ & $\begin{array}{l}\text { Breve revisión bibliográfica acerca de la actividad física practicada a finales del } \\
\text { S. } X V \text { y mediados del } S \text {. XVI. }\end{array}$ \\
\hline & Objetivos didácticos & Delimitación de los objetivos didácticos perseguidos. \\
\hline & Contenidos & $\begin{array}{l}\text { Especificación de los contenidos abordados, con especial atención a los juegos de } \\
\text { precisión. }\end{array}$ \\
\hline & $\begin{array}{l}\text { Aspectos } \\
\text { metodológicos }\end{array}$ & Aclaración de los estilos de enseñanza empleados en su puesta en práctica. \\
\hline & $\begin{array}{c}\text { Descripción y } \\
\text { temporalización }\end{array}$ & Número de sesiones y contenidos trabajados en cada una de ellas. \\
\hline & Evaluación & Evaluación empleada (qué, cuándo y cómo evaluar) \\
\hline & $\begin{array}{l}\text { Atención a la } \\
\text { diversidad }\end{array}$ & Planteamientos alternativos para la atención a acnee. \\
\hline & Interdisciplinariedad & Áreas que han contribuido a la elaboración de la Unidad Didáctica. \\
\hline \multicolumn{2}{|r|}{$\begin{array}{l}\text { Resultado del trabajo y } \\
\text { difusión }\end{array}$} & $\begin{array}{l}\text { Resumen y exposición fotográfica de la aplicación práctica, así como de su } \\
\text { difusión posterior. }\end{array}$ \\
\hline
\end{tabular}

\section{Introducción a la Unidad Didáctica}

Esta Unidad Didáctica, denominada «Aplicación de los juegos del medievo en las clases de Educación Física en laESO», está pensada para llevarse a cabo con alumnos ${ }^{1}$ de segundo curso de E.S.O., pero pensamos que con alguna ligera modificación podría trabajarse en cualquier curso de la etapa.

La gran mayoría de los juegos y tareas propuestas tendrán como denominador común la necesidad de demostrar precisión o puntería (coordinación óculo-manual) para desarrollarlos satisfactoriamente.

No se tratará tanto de jugar tal y como antes se jugaba, sino de practicarlos como ahora se conocen y desarrollan e, incluso, adaptarlos a nuestros intereses y necesidades. Eso sí, todo ello bajo el prisma histórico y la trascendencia del final del reinado de Isabel I.

Hemos empleado la transcripción que realizan Álvarez, García y Zapico (1997) del «Libro del Ejercicio Corporal y de sus provechos» de Cristóbal Méndez, fechado en el año 1553, para seleccionar los juegos y prácticas que más adelante llevaremos a cabo con los alumnos ${ }^{2}$. Por tanto, aclaramos que este documento será la fuente principal de la que surgirán muchas de nuestras propuestas. De hecho, tal y como se asegura en la edición consultada, es el primer autor del mundo que imprimía un texto sobre las virtudes del ejercicio físico.

\section{La actividad física en la época de Isabel I (1451-1504)}

En primer lugar, el humanista y pedagogo Juan Luis Vives (14921540), en su obra «De Tradentis Disciplinis» (1530) señalaba en un capítulo dedicado a la Educación Física de los jóvenes -aunque no en estos términos- prácticas y juegos de aquel momento: carreras, largas marchas, juegos, juego de pelota y de bolos, lucha y abatidas. El objetivo de todas ellas no era otro que evitar el embrutecimiento del cuerpo y vivificarlo, reponiendo y tonificando a su vez la mente ${ }^{3}$.

En 1553 Cristóbal Méndez escribe el «Libro del Ejercicio Corporal y de sus provechos». Se trata de un estudio sobre el ejercicio y los juegos propios de su época, con tintes filosóficos y médicos. Conviene aclarar que no es un tratado de Educación Física, pero perfectamente puede servir para hacernos una idea de los juegos, divertimentos y prácticas de aquella época.
De su lectura se desprende que, como así lo aclara en multitud de ocasiones el autor, el libro se circunscribe a Andalucía. No obstante, suponemos que la actividad fisica y juegos que allí se practicaban eran similares a los realizados en la vasta geografía que abarcaba el reino de Isabel I. De hecho, alguna provincia andaluza ya formaba parte de dicho reino (Sevilla, Córdoba, Jaén,...), a las que se sumó Granada tras su conquista en el 1492.

Cristóbal Méndez cita multitud de actividades, juegos y deportes de la época sin entrar a detallar cómo se jugaban, sus reglas o normas ${ }^{4}$, de ahí que nosotros hayamos hecho nuestra propia interpretación y adaptación de los mismos.

Además, estos autores corroboran la existencia de estos juegos y prácticas físicas con otros textos y literatura de la época ${ }^{5}$.

Nosotros hemos hallado en la obra La Celestina, de Fernando de Rojas-conforme a la edición de 1514-alusiones a actividades y prácticas físicas como la caza de altanería, justas, juegos de naipes y ajedrez. Además hay una breve reseña al juego del tablado en el que, según una nota aclaratoria del autor, había que derribar a pedradas un cántaro puesto en un tablado ${ }^{6}$, acepción bien distinta de la versión más extendida que recoge la Real Academia Española (2001) (RAE a partir de ahora) en la que el tablado era un armazón o castillete elevado contra el que se arrojaban lanzas con el fin de derribarlo o desbaratarlo.

Asimismo, hay novelas actuales que giran en torno a la reina Isabel I en las que se nombran juegos y entretenimientos como el juego del tablado, torneos, justas y celebraciones de cañas ${ }^{7}$. Por lo que respecta a esta última, se trataba de una fiesta a caballo en la que diferentes equipos se lanzaban recíprocamente unas cañas que no eran otra cosa que unas varitas de caña de unos seis palmos, derechas y limpias. El primero de sus cañutos se solía rellenar para hacerla más pesada y apta para el lanzamiento ${ }^{8}$.

Por otra parte, hemos constatado que el juego de los bolos suele ser del que más información escrita hay, encontrándose vestigios de su uso entre los egipcios, romanos y celtas ${ }^{9}$. Incluso hay algunos estudios que nos aclaran que este tipo de prácticas fueron sometidas a prohibiciones en aquella época ${ }^{10}$.

Finalmente, hemos hallado datos sobre un sistema de sorteo o echar suertes que también nos será de utilidad. En el Retablo mayor de la Catedral Vieja de Salamanca ${ }^{11}$, fechado entre $1445-1446$, se puede apre- 
ciar como varios soldados rodean a otro que sostiene en su mano tres pajas. Se trata del juego echar a pajas ${ }^{12}$, empleado para esclarecer el orden de participación de los jugadores en función del tamaño de la pajita extraída de la mano de otro compañero. También puede servir para ver quien elige juego o compañeros de equipo.

\section{Objetivos didácticos} de:

Al finalizar esta Unidad Didáctica los alumnos han de ser capaces

1. Conocer la figura y reinado de Isabel I.

2. Conocer las prácticas físicas y/o corporales relacionadas con el contexto de la Educación Física que tenían lugar durante dicha época, con especial atención a las de precisión.

3. Aprender y practicar juegos y actividades de precisión (coordinación óculo-manual) que perduran en su esencia desde la época de Isabel I, y que actualmente también tienen cabida en nuestra comunidad socio-educativa.

4. Adaptar y reciclar materiales para el desarrollo de juegos y tareas relacionados con este tipo de actividades de coordinación óculomanual.

5. Reconocer y valorar como parte de nuestro patrimonio histórico-cultural autonómico la época del reinado de Isabel I.

6. Utilizar los juegos y actividades de precisión como medio de catarsis y posible preparación para otras prácticas (incluso deportivas).

7. Ser capaz de practicar estos juegos en un contexto extraescolar con sus familiares y amigos.

8. Investigar sobre prácticas, juegos o actividades que empleaban nuestros mayores como medio de diversión y ocupación del tiempo libre, recuperándolas del exilio al que están siendo sometidas en nuestra sociedad.

9. Aceptar y emplear la competición con una actitud meramente motivadora, sin desarrollar ningún tipo de rivalidad hacia el oponente.

10. Adquirir autonomía en la elaboración y desarrollo de competiciones, valorando por encima de todo la propia participación y el respeto por la esencia misma de los juegos realizados.

\section{Contenidos}

1. Características generales del reinado de Isabel I.

2. Prácticas físicas y juegos de la época.

3. Cualidades fisicas.

4. Evolución de dichas prácticas hasta el momento actual.

5. Coordinación dinámica general y segmentaria.

6. Práctica de juegos de precisión:

- Jabalina: Además de la modalidad de lanzamiento de precisión, también emplearemos la de distancia.

- Tablado: En las dos versiones aludidas anteriormente: derribar cántaros y castilletes.

- Herrón: Aunque Cristóbal Méndez ${ }^{13}$ considera que el herrón es el mismo juego que el tejo, somos partidarios de emplear la versión que señala que el herrón es un juego que consistía en meter en un clavo hincado en el suelo unos discos de hierro con un agujero en el centro (RAE, 2001).

- Lanzamiento de herradura: Lo diferenciamos del anterior en el material de juego empleado: en este caso son las propias herraduras (hierros semicirculares) que se clavan en los cascos de las caballerías.

- Rana, bolos, calva, tanga y billar romano: Estos son algunos de los juegos y deportes considerados autóctonos y tradicionales de Castilla y León (Ley 2/2003).

- Dardos: Fabricados con pajitas de beber lastradas en uno de sus extremos con esparadrapo o similar para posibilitar un mejor vuelo. Las dianas se elaboran con cartones a los que se les realizan diferentes tipos de huecos por los que meter los dardos.

- Tejo: Cada uno de los juegos en que se emplea un tejo -pequeña teja o cosa semejante-(RAE, 2001). En concreto empleamos el juego de la raya o tirar a raya ${ }^{14}$ consistente en lanzar el tejo desde una distancia con la intención de situarlo lo más cerca posible de una raya dibujada o marcada en el suelo. El orden de participación se establece en función de la proximidad de la moneda o piedra a la raya.

- Cañas: Usaremos a modo de cañas los dardos fabricados con pajitas. Como escudos se utilizarán las palas de madera. Habrá diferentes posibilidades de juego: persecuciones, puntería, repeler los dardos sin mover los pies...

- Argolla: Juego cuyo principal instrumento es una argolla de hierro que, con una espiga o punta aguda que tiene, se clava en la tierra de modo que pueda moverse fácilmente alrededor, y por lo cual se han de hacer pasar unas bolas de madera que se impelen con palos cóncavos (RAE, 2001).

- Vilorta: Juego que consiste en lanzar por el aire, con ayuda del vilorto (palo grueso que termina por una de sus puntas en forma de aro, y encordelado a modo de raqueta), una bola de madera que ha de pasar a través de la fila de pinas o estacas colocada entre los dos bandos de jugadores (RAE, 2001). Se trata de un juego que bien podría decirse que se parece al lacrosse ${ }^{15}$.

- Chueca: Juego que se hace poniéndose los jugadores unos enfrente de otros en dos bandas iguales, procurando cada uno que la chueca (bolita pequeña con que se juega), impelida con palos por los contrarios, no pase la raya que señala su término(RAE). En definitiva, juego similar al hockey actual.

7. Normas, reglas y formas de los juegos y actividades de precisión.

8. Elaboración y reciclaje de material.

9. Valoración y reconocimiento del patrimonio histórico y cultural de Castilla y León, especialmente el relacionado con las actividades físicas autóctonas.

10. Conocimiento del uso y valor del juego tradicional por parte de nuestros mayores, y recuperación de dichos juegos.

\section{Aspectos metodológicos}

A lo largo de las sesiones emplearemos básicamente dos estilos de enseñanza: la asignación de tareas y el descubrimiento guiado.

En la Asignación de tareas la práctica se introduce en pequeñas tareas que van a permitir una mayor individualización, por lo que va más encaminada a la adquisición por parte del alumno de unos correctos patrones de ejecución y un aprendizaje rápido y adecuado para cada uno de los juegos y tareas propuestas. Por otro lado, con el Descubrimiento guiado pretendemos guiar a los alumnos a lo largo de una serie de problemas en los que se explorarán las soluciones y se tomarán decisiones para encontrar la que parezca más apropiada, provocando así la indagación e implicación más profunda del alumno en la actividad planteada.

\section{Descripción y temporalización}

Esta Unidad Didáctica está ideada para aplicarse a lo largo del tercer trimestre. En todas las sesiones se comenzará con una explicación previa en la que se detallarán los objetivos a conseguir, los contenidos que se trabajarán, cómo nos agruparemos, y los juegos y tareas que se practicarán. Por otra parte, al término de cada sesión se dedicará un tiempo para el conocimiento de los resultados, intercambio de experiencias, comentarios y observaciones a la práctica, trabajo del cuaderno de clase, etc.

A partir de una propuesta inicial de trabajo en el final de cualquier clase, proponemos una secuencia de desarrollo en 13 sesiones (Gráfico 1)

\section{Evaluación}

Entendemos la evaluación como un proceso de recogida de información para su posterior análisis, con la consiguiente toma de dediciones en función de los resultados obtenidos ${ }^{16}$. 


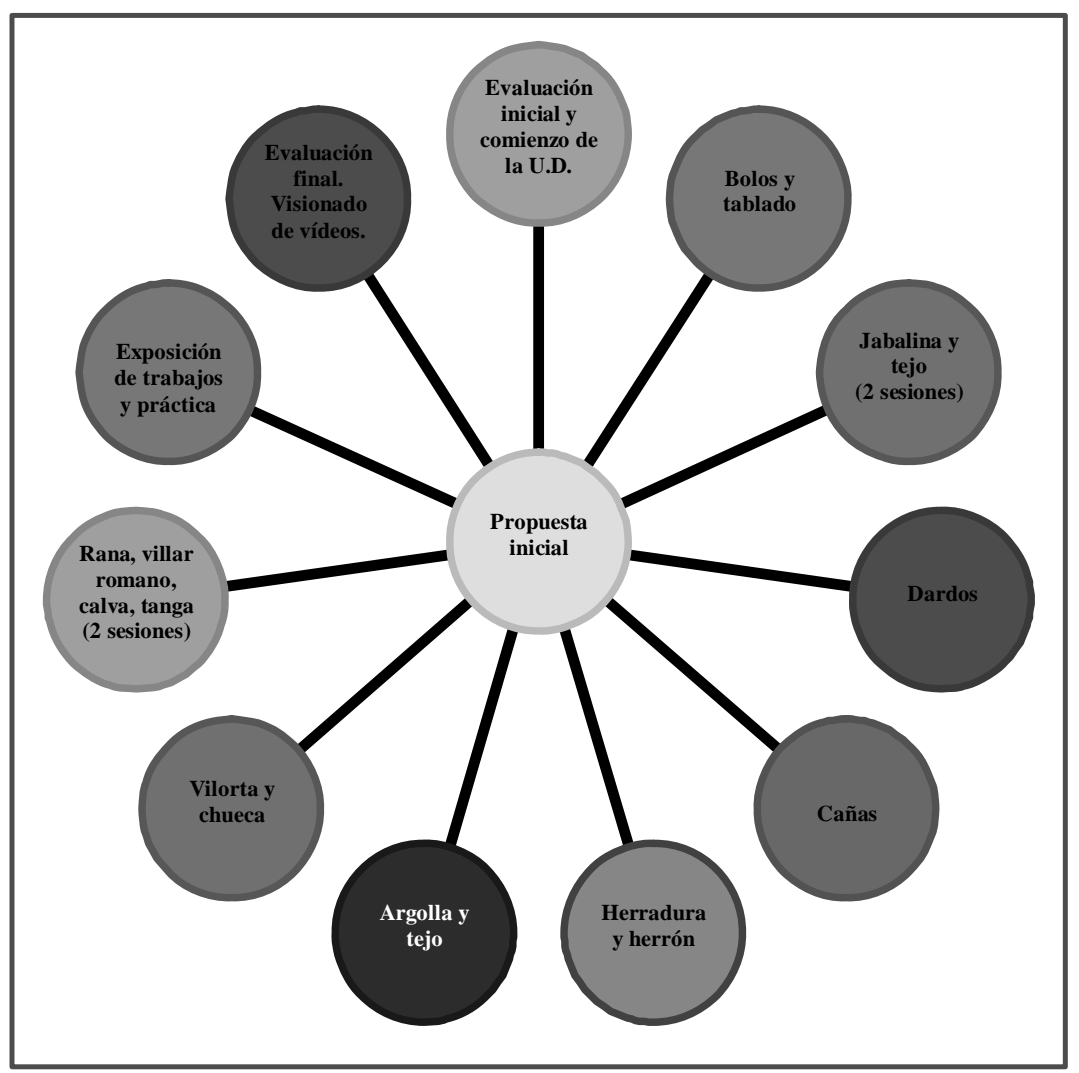

Gráfico 1: Secuencia de desarrollo de las sesiones.

\subsection{Qué evaluar}

\section{Evaluación del alumno}

Como sabemos, los criterios de evaluación expresan un grado de aprendizaje determinado por la capacidad que se trata de evaluar y el contenido a través del cual se desarrolla ${ }^{17}$. Nosotros emplearemos los siguientes: físicas.

- Conocer la figura y época de Isabel I en relación a las actividades

- Participar en los juegos y actividades lúdicas previstas.

- Realizar correctamente el cuaderno de clase.

- Investigar y recopilar juegos y actividades lúdicas practicadas por generaciones pasadas.

- Realizar las diversas formas de lanzamiento requeridas por las actividades propuestas.

- Crear recursos para el juego a partir de material reciclado.

- Conocer las actividades y juegos tradicionales, y valorarlos como identificadores del patrimonio cultural de Castilla y León.

Evaluación del proceso

La evaluación del proceso se puede definir como el examen intencional y sistemático de la propia acción didáctica ${ }^{18}$. Por tanto, habrá que evaluar la validez de los objetivos, la adecuación de los contenidos, la temporalización propuesta, la metodología aplicada, los recursos didácticos empleados, y las técnicas e instrumentos de evaluación (metaevaluación).

\section{Evaluación del profesor}

La actuación del profesor es clave en todo el proceso de enseñanzaaprendizaje, siendo él mismo el encargado de analizarla pues es quien más información tiene al respecto. No obstante, la aportación de los compañeros del área y de los propios alumnos puede ayudar a aumentar la calidad y cantidad de información el profesor.

\subsection{Cuándo evaluar}

\section{Evaluación inicial}

La evaluación inicial debe estar al servicio tanto del profesor como del propio alumno, ya que al primero le aporta datos sobre el cómo y el dónde están los segundos en relación a unos contenidos, y a éstos les sitúa ante nuevos retos y aprendizajes.

Incluso antes de comenzar la Unidad Didáctica, en una sesión anterior, les pediremos que escriban en sus cuadernos lo que esperan aprender, si conocen algún juego en el que haya que lanzar objetos, etc.

Evaluación continua o formativa

El profesor, a través de sus propias anotaciones, recogida de observaciones y comentarios de los alumnos, etc., tendrá información suficiente como para permitirle conducir el proceso de enseñanza-aprendizaje, adoptar los cambios que estime oportunos, informar a los alumnos sobre sus logros y la resolución de las dificultades,...

Evaluaciónfinal

Coincidiendo con el final de la Unidad Didáctica realizaremos una evaluación sumativa. Ésta no sólo debe permitir asignar calificaciones a los alumnos, sino que además tiene que servir para analizar el nivel de éxito o fracaso de todo el proceso educativo y para poner los medios necesarios que permitan reconducir o plantear de nuevo la situación. También puede ser utilizada como punto de partida para el establecimiento de nuevos aprendizajes o nuevas intenciones educativas, y sienta las bases de futuras evaluaciones iniciales.

No obstante, es inevitable tomar decisiones acerca de la calificación que los alumnos deben tener, la cual debe «reflejan» el logro de sus aprendizajes y el trabajo realizado. Para ello, aplicaremos los siguientes criterios de calificación:

- Trabajo de investigación. $15 \%$.

- Trabajo desarrollado en clase y actitudes $\quad 55 \%$.

- Cuaderno de clase $30 \%$.

\subsection{Cómo evaluar}

Uno de los retos que nos hemos planteado es el de utilizar mecanismos que propicien la participación del alumno en su proceso de evaluación (autoevaluación). Por ello, hemos diseñado y empleado varios instrumentos:

Ficha de autoevaluación

En esta ficha, entregada al alumno al comienzo de la Unidad Didáctica, le proponemos que conteste a una serie de cuestiones (siempre las 


\section{Jornadas Regionales de Experiencias Didácticas sobre Isabel I y su época}

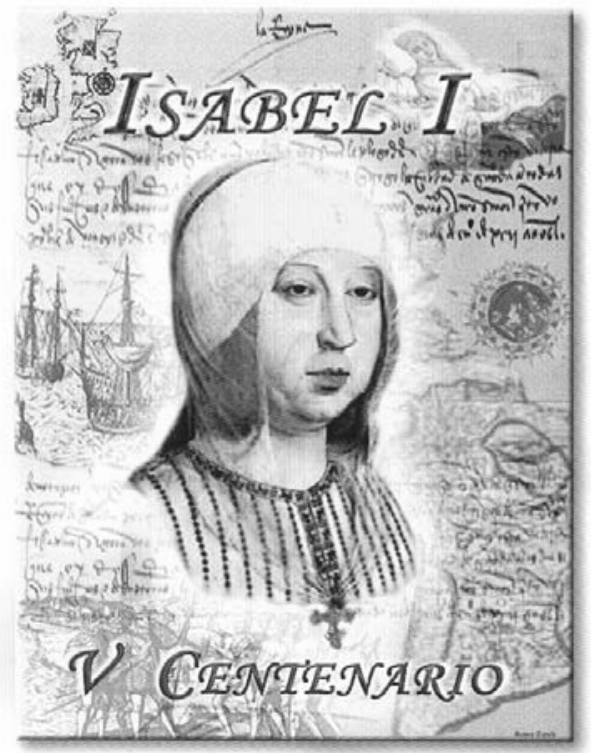

Castillo de la Mota - 28 y 29 de junio Medina del Campo $\cdot 2004$
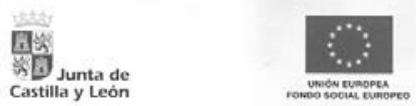

mismas) para cada una de las sesiones que la componen. Se trata de responder empleando una escala numérica, del 1 al 10, siendo el 1 lo más bajo, negativo, etc., y el 10 todo lo contrario.

En aquellas preguntas o afirmaciones que la respuesta emitida esté por debajo del 5, el alumno deberá de razonar y explicar en su cuaderno los motivos o razones de dicha respuesta.

Mediante esta ficha de autoevaluación el alumno realiza una valoración y reflexión diaria de todo lo que acontece en torno a su propia práctica, llegando a ser plenamente consciente de sus avances y, sobre todo, de sus puntos débiles y dificultades, y la manera de superarlos.

Trabajo de investigación

Desde el principio de la Unidad Didáctica advertimos a los alumnos la obligatoriedad de realizar un trabajo que verse sobre los juegos y pasatiempos (con un claro componente físico o de movimiento) que practicaban hace años sus familiares más mayores. Son varias las metas que podemos alcanzar:

- Conocer los juegos, tareas y divertimentos practicados por nuestros mayores.

- Reconocer el valor cultural popular que llegaron a tener esos juegos.

- Propiciar que los adolescentes se relacionen con los familiares de más edad y que éstos colaboren con ellos en su proceso de enseñanzaaprendizaje, haciéndoles sentir parte importante y útil en la sociedad.

- Recuperar juegos olvidados y disfrutar de ellos.

Cuaderno de clase del alumno

Se trata de un cuaderno en blanco en el que el alumno irá recogiendo lo más significativo de las sesiones, anotaciones realizadas por el profesor o compañeros, actividades y juegos realizados,..., además de ser un lugar en el que plasmar su opinión, intereses, dudas, sentimientos y poder tener así una vía más personal de comunicación con el profesor ${ }^{19}$.

Informe de evaluación

Al final del último día dedicado a la Unidad Didáctica, como colofón al trabajo realizado durante las sesiones, entregaremos a cada alumno un informe de evaluación.

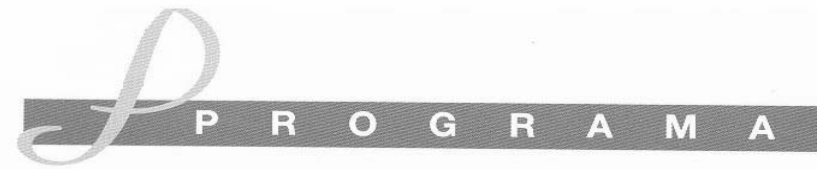

Día 28 de junio de 2004 :

\begin{tabular}{|c|c|}
\hline 9:30 a 10 h: & Recogida de documentación. \\
\hline 10 a 10:30 h: & Inauguración de las Jornadas. \\
\hline$: 30$ a $11 \mathrm{h:}$ & Descanso. \\
\hline 11 a $12: 30 \mathrm{~h}:$ & $\begin{array}{l}\text { Conferencia Inaugural: "Isabel, Infanta y Princesa" por Dra. Isabel } \\
\text { del Val. }\end{array}$ \\
\hline $12: 30 \mathrm{~h}:$ & $\begin{array}{l}\text { "Elaboración de materiales multimedia sobre el v } \\
\text { Centenario de la muerte de Isabel la Católica". } \\
\text { Autor: José Manuel Ara Encinar. }\end{array}$ \\
\hline 13:00 h: & $\begin{array}{l}\text { "Ámbitos culturales en tiempos de Isabel I de Castilla". } \\
\text { Autores: Joana Boscá Vidal / Félix Ferrer García. }\end{array}$ \\
\hline 13:30 h: & $\begin{array}{l}\text { "La Reina Isabel la Católica". } \\
\text { Autora: Paula Ruano Díaz. }\end{array}$ \\
\hline 16:00 h: & $\begin{array}{l}\text { "Isabel I de Castilla: la reina que creyó en un sueño". } \\
\text { Autora: Candelas Gómez Sánchez. }\end{array}$ \\
\hline $16: 30 \mathrm{~h}:$ & $\begin{array}{l}\text { "Isabel I de Castilla: mujer y reina". } \\
\text { Autores: Tomás Fernández Fdez. / Julián Fdez. Martín / Ma del } \\
\text { Carmen Glez. Fuentes / Ricardo Eugenio Tirados Blanco. }\end{array}$ \\
\hline $17: 30 \mathrm{~h}:$ & $\begin{array}{l}\text { "Isabel I de Castilla y Dueñas". } \\
\text { Autores: Pedro Díez Montes / Félix Pinedo Paredes. }\end{array}$ \\
\hline 18:00 h: & $\begin{array}{l}\text { "Mito, leyenda y realidad de una Reina". } \\
\text { Autora: Sonsoles Montejo González. }\end{array}$ \\
\hline 18:30 h: & $\begin{array}{l}\text { "Influencia del reinado de Isabel la Católica en las Artes } \\
\text { Aplicadas actuales". Autora: Luisa Carmen del Río Díaz. }\end{array}$ \\
\hline 19:00 h: & $\begin{array}{l}\text { "Itinerario castellano de una Reina". } \\
\text { Autor: Alberto Gómez Nieva. }\end{array}$ \\
\hline 19:30 h: & $\begin{array}{l}\text { "Danzas en la época de Isabel I de Castilla". } \\
\text { Autora: Rosa M Carnicero Ahedo. }\end{array}$ \\
\hline 20:00 h: & $\begin{array}{l}\text { "Los Juegos en la época de la Reina Isabel I". } \\
\text { Autor: Julio César Rodríguez de la Cruz. }\end{array}$ \\
\hline 22:00 h: & $\begin{array}{l}\text { Concierto de voz y guitarra: "En el Jardín de la Reina. Cantos } \\
\text { Judíos y Cristianos". Dña. Ma José Cordero. Profesora Superior } \\
\text { de Piano y D. Juan José Collado. Profesor Superior de Guitarra. } \\
\text { Conservatorio Profesional de Música "Cristóbal Halffter"de } \\
\text { Ponferrada. }\end{array}$ \\
\hline
\end{tabular}

A grandes rasgos, consiste en una serie de preguntas abiertas sobre diferentes ámbitos o aspectos que intervienen en todo el proceso: profesor, él mismo, compañeros, materiales, cuaderno de clase, evaluación,..., y que deben ser contestadas fuera de clase. Será anónimo para crear un clima de confianza en el alumno que le haga ser completamente sincero, sin temor a represalias o consecuencias de ningún tipo. Se devolverá completado en la siguiente clase de Educación Física.

Al margen de los instrumentos utilizados por el alumno, el profesor empleará básicamente dos:

\section{Cuaderno de notas del profesor}

No se trata de un cuaderno de notas (calificaciones) en el sentido literal, sino que más bien es un documento en el que recogemos comentarios de nuestros alumnos, observaciones, sucesos, propuestas,..., que hayan tenido lugar durante las sesiones. Pero sobre todo plasmaremos nuestras reflexiones, fruto del análisis de las prácticas. Las anotaciones podrán tener carácter individual, referidas a un alumno en concreto, $\mathrm{o}$ colectivo, respecto de todo el grupo.

Ficha de observación del alumno

Hemos diseñado una ficha de observación con varios ítems acordes a la propuesta didáctica que estamos llevando a cabo. Cada alumno tendrá la suya, y diariamente la rellenaremos contestando con un sí o un no. Obviamente, esta ficha nos aporta más información sobre cómo ha sido el proceso de enseñanza-aprendizaje del alumno.

\section{Atención a la diversidad}

En nuestras clases podemos encontrarnos con una variedad de alumnos en lo que a sus características, intereses, motivaciones,..., se refiere. A todos ellos debemos de atenderlos por igual, adoptando y adaptando las actividades que sean necesarias y que se ajusten a todas esas peculiaridades ${ }^{20}$.

El caso más extremo de atención a la diversidad que nos podemos encontrar es el de los alumnos con necesidades educativas especiales asociadas a cualquier tipo de discapacidad. Queremos aclarar que para nosotros todos los alumnos tienen unas especiales necesidades educati- 
vas debidas, entre otras cosas, a la individualidad de cada uno. Por eso, a estos «casos extremos» consideramos más adecuado denominarlos alumnos con dificultades de aprendizaje asociadas a discapacidad.

Esbozamos a continuación una serie de propuestas encaminadas a orientar al profesor en la aplicación de medidas o adaptaciones para esos alumnos con dificultades de aprendizaje:

- Modificar distancias de lanzamiento.

- Variar la posición de lanzamiento (sentado, pata coja, etc.)

- No sólo utilizar el brazo o mano dominante.

- Posibilidad de lanzar dificultando (o suprimiendo incluso) la vista: ayuda de guías.

- «Lanzar» con el pie en vez de con la mano.

- Modificar tamañoy/o disposición de la diana u objeto a dar (grandes globos en vez de botes, bolos más juntos, etc.)

- Variar tamaño, peso y/o forma de los elementos lanzados (monedas en vez de «chinos» en la rana, pelotas-globos en vez de bolas, globos rellenos en vez de bolas para el billar romano, pelotas de playa, etc.)

- Proponer algún tipo de juego o deporte adaptado específico de alumnos con discapacidad: la boccia.

\section{Interdisciplinaridad}

Obviamente, la colaboración con otras áreas que forman parte del currículo de la Enseñanza Secundaria es siempre conveniente y necesaria, por lo que no debemos de trabajar de forma aislada y cerrada al resto de las materias, implicando a cuantos más elementos del sistema educativo podamos.

Concretamente, las áreas con las que hemos mantenido contactos para la elaboración de este trabajo han sido: Tecnología (confección de material alternativo), Ciencias Sociales (acceso a información y ubicación histórica), y Lengua y Literatura Castellana (facilitación de obras y textos de lectura).

\section{Resultado del trabajo y difusión}

Como ya apuntábamos al inicio del artículo, el resultado que obtuvimos con el desarrollo de la U.D., tanto en lo concerniente a la propia práctica como a la elaboración de los materiales, dio unos frutos más valiosos de lo inicialmente esperados.

Su puesta en práctica se llevó a cabo con grupos de $2^{\circ}$ de E.S.O., algunos de los cuales con muy bajo interés hacia cualquier tipo de actividad física e, incluso, con alumnado inmigrante. La mayoría de los juegos despertaron un interés inusual por querer participar y por elaborar los materiales de juego. La implicación, entusiasmo y disponibilidad fueron la tónica general, sobre todo en los juegos más desconocidos o poco usuales en nuestras clases (dardos, cañas, jabalina, tablado...). Además, como colofón a la U.D. pudimos realizar una excursión a Medina del Campo, localidad en la que ese año se realizaron multitud de eventos culturales en relación a este personaje histórico.

Finalmente, nuestro trabajo fue seleccionado por el Centro de Formación e Innovación Educativa de nuestro ámbito para que fuese expuesto en las jornadas regionales de experiencias didácticas sobre Isabel I que tuvieron lugar en Medina del Campo (ilustración 1 y 2)

\section{Bibliografía}

Álvarez, E., García, C. y Zapico, J.M. (1997). Cristóbal Méndez y su obra-1553. Transcripción moderna y comentarios. Málaga: Instituto Andaluz del Deporte.

Álvarez del Palacio, E. (2003). Juego, educación física y deporte en el Renacimiento español. En Rodríguez Rodríguez, L.P., Compendio histórico de la actividad física yel deporte (pp. 171-211), Barcelona: MASSON.

Andrés Martín, F. (1990). Juegos y Deportes Autóctonos. Salamanca: Centro de Cultura tradicional de la Diputación de Salamanca.

Bernardo, J. y Calderero, J.F. (2000). Aprendo a investigar en Educación. Madrid: RIALP.
Blández Ángel, J. (2000). La investigación-acción: Un reto para el profesorado. Zaragoza: INDE.

Blázquez, D. (1997). La evaluación de la educación física. Evaluación del proceso de enseñanza-aprendizaje. En Hernández, J., Salud, Deportey Educación (pp. 375-396). Gran Canaria: ICEPSS Editores.

De Irisarri, A. (2001a). Isabel, la Reina: Las hijas de la luna roja. Barcelona: Círculo de Lectores.

De Irisarri,A. (2001b). Isabel, la Reina: El tiempo de la siembra. Barcelona: Círculo de Lectores.

De Rojas, F. (1983, Edición de Bruno Mario Damiani). La Celestina. Madrid: Cátedra.

Real Academia Española (2001). Diccionario de la lengua española (22a. ed.). Madrid: Espasa-Calpe.

Ley $2 / 2003$, de 28 de marzo, del Deporte de Castilla y León (BOCyL 04-04-2003).

M.E.C. (1992). Secundaria obligatoria: Educación Física. Madrid: MEC.

Moreno Palos, C. (1992). Juegos y Deportes tradicionales en España. Madrid: Alianza Deportes.

Nuevo Diccionario Enciclopédico Universal Durvan (1992). Madrid: Club Internacional del Libro.

Ríos, M. y Fraile,A. (2003). La atención a la diversidad como necesidad educativa. La inclusión del alumnado con discapacidad: una escuela para todos y todas. En Fraile, A. (Dir.), Actividad física y salud. Educación secundaria (pp. 37-45). León: Junta de Castilla y León.

Rodríguez de la Cruz, J.C. (2006). El lacrosse como contenido deportivo de la Educación Física en Secundaria. DEPORTES.DIP, 20, Separata Técnica $\mathrm{N}^{\circ} 4$.

Rodríguez de la Cruz, J.C. y Lera Corredera J.M. (2006, septiembre). El cuaderno de clase del alumno/a como instrumento de evaluación y vehículo de comunicación en la Educación Física. Lecturas: Educación Física y Deportes [en línea], № 100. Disponible en: http:// www.efdeportes.com/efd100/cuad.htm

Rodríguez Rodríguez, L.P. (2003). Estudio y comentarios sobre El libro del ejercicio corporal y de sus provechos (1553), de Cristóbal Méndez. En Rodríguez Rodríguez, L.P., Compendio histórico de la actividad física y el deporte (pp. 213-268). Barcelona: MASSON.

Sánchez, M. (1982). Vida popular en Castilla y León a través del arte (edad media). Valladolid: ÁMBITO.

Sánchez Pinto, C. (2005). Los jubilosos juegos jubilados. Valladolid: ADRIMO.

Veleda Vallelado, M.J. (Coord.) (1998). Reglamentos de los Deportes Autóctonos de Castillay León. Valladolid: Caja España.

\section{(Notas)}

${ }^{1}$ Emplearemos el término alumno para referirnos por igual a alumnos y a alumnas. Igualmente sucederá con el término profesor.

${ }^{2}$ Además han sido de gran apoyo y complemento Álvarez (2003) y Rodríguez (2003).

${ }^{3}$ Vid.: Moreno (1992); Veleda (1998).

${ }^{4}$ Álvarez et al. (1997: 60).

${ }^{5}$ Ibidem: 58-61.

${ }^{6}$ Edición de Bruno Mario Damiani (1983).

${ }^{7}$ Vid.: De Irisarri (2001a; 2001b).

${ }^{8}$ Cfr:: Nuevo Diccionario Enciclopédico Universal Durvan (1992).

${ }^{9}$ Vid.: Andrés (1990: 53).

${ }^{10}$ Vid.: Veleda (1998: 87) y Moreno (1992: 74).

${ }^{11}$ Vid.: Sánchez (1982: 114).

${ }^{12}$ Vid.: Sánchez (2005: 33).

${ }^{13}$ Vid.: Álvarez et al. (1997: 199).

${ }^{14}$ Vid.: Sánchez (2005: 35).

${ }^{15}$ Vid.: Rodríguez de la Cruz (2006).

${ }^{16}$ Cfr: MEC (1992)

${ }^{17}$ Cfr:: Blázquez (1997).

${ }^{18}$ Ibdidem.

${ }^{19}$ Para conocer con más profundidad este instrumento de trabajo recomendamos la lectura de Rodríguez y Lera (2006)

${ }^{20}$ Sobre este tema recomendamos la lectura de Ríos y Fraile (2003). 